\title{
Dysfunction induced by ischemia versus edema: Does edema matter?
}

\author{
Tanya L. Butler, BSc, PhD, ${ }^{\text {a,b }}$ Jonathan R. Egan, MBBS, FRACP, ${ }^{\text {a,b }}$ Fabian G. Graf, ${ }^{\text {a }}$ Carol G. Au, BSc, a,b \\ Aisling C. McMahon, PhD, ${ }^{d}$ Kathryn N. North, MD, FRACP, ${ }^{b, c}$ and David S. Winlaw, MD, FRACS ${ }^{a, b, e}$
}

Objectives: Recovery from pediatric cardiac surgery is affected by ischemia-reperfusion injury, cardiac edema, and in some cases a low cardiac output syndrome. Although association has been made between the development of edema and dysfunction, modeling is confounded by intercurrent injurious stimuli that also cause cardiac edema and dysfunction. We tested whether a true causal relationship exists between edema and cardiac dysfunction.

Methods: We induced either ischemia or edema alone in isolated cardiomyocytes and whole Langendorff-perfused hearts. Function was measured as shortening dynamics and developed pressure, respectively.

Results: Ischemic injury impaired function in both cardiomyocytes and whole hearts. Isolated cells showed significant reduction in peak shortening and departure and relaxation velocities. Whole hearts displayed severely reduced developed pressures. Hyposmotic solution forced cardiomyocytes to swell to $7 \%$ greater than their normal size. No significant effect on shortening was seen. Similarly, Langendorff-perfused hearts were induced to take on $3 \%$ more water than control-perfused hearts and $9 \%$ more water than nonperfused hearts. This additional water was associated with mild dysfunction.

Conclusions: We demonstrate the capacity of the heart to tolerate edema greater than that seen in clinical settings without residual effect. Ischemia results in ongoing contractile dysfunction of both isolated cardiomyocytes and whole hearts. We conclude that dysfunction resulting from edema in ex vivo cardiac models is mild and suggest review of the importance given to edema-mediated dysfunction after cardiac surgery.

Supplemental material is available online.

Development of cardiac dysfunction is a significant clinical concern after cardiac surgery with cardiopulmonary bypass and myocardial ischemia managed with cooling and preservation solutions. As many as $20 \%$ of pediatric patients undergoing surgical intervention to correct congenital heart defects experience a clinically significant low cardiac output syndrome in the first 6 to 24 hours of their recovery. Potential sources of myocardial damage that can affect function include direct myocardial trauma (eg, ventriculotomy), the systemic

\footnotetext{
From Kids Heart Research, ${ }^{\text {a }}$ The Children's Hospital at Westmead, Sydney, Australia; the Discipline of Paediatrics and Child Health, ${ }^{\mathrm{b}}$ Faculty of Medicine, University of Sydney, Sydney, Australia; the Neurogenetics Research Unit and the Institute for Neuromuscular Research, ${ }^{\text {c }}$ The Children's Hospital at Westmead, Sydney, Australia; the Vascular Biology Group, ${ }^{\mathrm{d}}$ ANZAC Research Institute, Concord RG Hospital, University of Sydney, Sydney, Australia; and Cardiothoracic Surgery, Adolph Basser Cardiac Research, The Children's Hospital at Westmead, Sydney, Australia.

This research was supported by the National Health and Medical Research Council of Australia (NHMRC Project grant 40271). J.R.E. and C.G.A. are supported by NHMRC Biomedical Research Scholarships (297113 and 358800), and D.S.W. is a National Heart Foundation of Australia Career Development Fellow.

Received for publication Aug 25, 2008; revisions received Nov 13, 2008; accepted for publication Dec 14, 2008.

Address for reprints: David S. Winlaw, MD, FRACS, Kids Heart Research, Locked Bag 4001, Westmead NSW 2145, Australia (E-mail: davidw@chw.edu.au).

J Thorac Cardiovasc Surg 2009;138:141-7

$0022-5223 / \$ 36.00$

Copyright (c) 2009 by The American Association for Thoracic Surgery

doi:10.1016/j.jtcvs.2008.12.008
}

inflammatory response to cardiopulmonary bypass, ischemia, and ischemia-reperfusion injury. ${ }^{1,2}$ Edema is also commonly included in this list of potential effectors because it is temporally associated with development of low cardiac output. ${ }^{3}$

Various studies have argued that edema is causal in the development of cardiac dysfunction. ${ }^{3}$ Both edema and cardiac dysfunction have a number of initiators and promoters, making a direct causal relationship difficult to prove. Ischemia, ${ }^{4}$ endothelial damage with capillary leak, ${ }^{5}$ and hypothermic cardioplegia $^{6}$ are all associated with the development of edema. However, these injuries are complex and sometimes interrelated in their effects. Thus a true causal relationship between edema and cardiac dysfunction remains to be established. We have recently examined this in a whole-animal model, ${ }^{7}$ reinforcing the need for in vitro dissection of these pathways.

Several studies using isolated cardiomyocytes ${ }^{6,8,9}$ and ex vivo whole hearts ${ }^{10}$ have been put forward as evidence for a causal relationship between edema and cardiac dysfunction. As with surgical models, coexisting factors, including ischemia and hyperkalemic cardioplegia, complicate interpretation. We aimed to specifically examine the effect of swelling on the function of isolated cardiomyocytes and whole hearts by using simple tonicity change to definitively determine the importance of edema to dysfunction. Ischemic injury was separately investigated.

\section{MATERIALS AND METHODS}

Experiments were approved by the Westmead Hospital Animal Ethics Committee. Animals received humane care in compliance with the National Health and Medical Research Council of Australia guidelines. 


\section{Abbreviation and Acronym \\ HEPES $=$ N-2-hydroxyethylpiperazine- \\ $\mathrm{N}$-2-ethanesulfonic acid}

\section{Isolation of Cardiomyocytes}

Wistar rats (200-350 g) were anesthetized with inhaled isoflurane. Sternotomy was performed, and the heart was excised and immediately placed in ice-cold Krebs buffer (Sigma, St Louis, Mo). After cannulation, hearts were perfused at 6 to $8 \mathrm{~mL} / \mathrm{min}$, with oxygenated solutions warmed to $37^{\circ} \mathrm{C}$. Perfusion for 5 minutes with Krebs buffer was followed by 5 minutes of perfusion with nominally calcium-free Tyrode's buffer containing 140 $\mathrm{mmol} / \mathrm{L} \mathrm{NaCl}, 5.4 \mathrm{mmol} / \mathrm{L} \mathrm{KCl}, 1 \mathrm{mmol} / \mathrm{L} \mathrm{MgCl}_{2}, 5 \mathrm{mmol} / \mathrm{L} \mathrm{N}$-2-hydroxyethylpiperazine-N-2-ethanesulfonic acid (HEPES), $20 \mathrm{mmol} / \mathrm{L}$ taurine, and $10 \mathrm{mmol} / \mathrm{L}$ glucose. Digestion was then achieved by means of perfusion for 20 minutes with Tyrode's buffer containing $1 \mathrm{mg} / \mathrm{mL}$ collagenase type II (Worthington Biomedical, Lakewood, NJ), $1 \mathrm{mg} / \mathrm{mL}$ hyaluronidase type I (Sigma), and $175 \mu \mathrm{mol} / \mathrm{L} \mathrm{CaCl}_{2}$. Hearts were removed from the apparatus, coarsely minced, and subjected to $3 \times 5$-minute rounds of digestion at $37^{\circ} \mathrm{C}$ with gentle mechanical agitation. Cells were filtered through a $180-\mu \mathrm{m}$ filter, pelleted by means of gravity, and washed in Tyrode's buffer containing increasing amounts of calcium to a final concentration of $1 \mathrm{mmol} / \mathrm{L}$.

\section{Isolated Cardiomyocyte Buffers}

Cardiomyocytes were placed in a custom chamber (IonOptix Corp, Milton, Mass) on an inverted microscope (Coherent Scientific, Hilton, Australia) and perfused at 1 to $2 \mathrm{~mL} / \mathrm{min}$, with buffers warmed inline (Cell MicroControls, Norfolk, Va) to $25^{\circ} \mathrm{C}$. Buffers for swelling studies were taken from the work of Ogura and colleagues ${ }^{11}$ with some modification and are summarized in Table E1. The primary buffer was Tyrode's buffer, containing $140 \mathrm{mmol} /$ $\mathrm{L} \mathrm{NaCl}, 5.4 \mathrm{mmol} / \mathrm{L} \mathrm{KCl}, 1 \mathrm{mmol} / \mathrm{L} \mathrm{MgCl} 2,5 \mathrm{mmol} / \mathrm{L}$ HEPES, $10 \mathrm{mmol} / \mathrm{L}$ glucose, and $1 \mathrm{mmol} / \mathrm{L} \mathrm{CaCl}_{2}$, which is referred to as Isotonic (1T). Tyrode's buffer in which $35 \mathrm{mmol} / \mathrm{L} \mathrm{NaCl}$ was replaced with $70 \mathrm{mmol} / \mathrm{L}$ sucrose was referred to as LowNa Isotonic, and Tyrode's buffer with $35 \mathrm{mmol} / \mathrm{L} \mathrm{NaCl}$ removed to make the solution hypotonic was referred to as $0.75 \mathrm{~T}$. This degree of tonicity is at the moderate end of previously described models ${ }^{11}$ and induces cardiomyocyte swelling to a degree that is broadly comparable with that experienced by infants after cardiopulmonary bypass. ${ }^{12,13}$ Buffers for ischemia experiments were taken from Lu and coworkers ${ }^{14}$ and contained $140 \mathrm{mmol} / \mathrm{L} \mathrm{NaCl}, 5.4 \mathrm{mmol} / \mathrm{L} \mathrm{KCl}, 1 \mathrm{mmol} / \mathrm{L} \mathrm{MgCl}_{2}, 5 \mathrm{mmol} / \mathrm{L}$ HEPES, 1 $\mathrm{mmol} / \mathrm{L} \mathrm{CaCl}_{2}$, and $2.5 \mathrm{mmol} / \mathrm{L} \mathrm{Na}$-lactate. Isotonic, LowNa Isotonic, and $0.75 \mathrm{~T}$ buffers were adjusted to $\mathrm{pH} 7.4$ and oxygenated, whereas the ischemic buffer was adjusted to $\mathrm{pH} 6.8$ and bubbled with $100 \% \mathrm{~N}_{2}$.

\section{Isolated Cardiomyocyte Functional Analysis}

Cardiomyocytes were loaded with Fura-2-AM (Invitrogen, Carlsbad, Calif) by means of incubation in a $5 \mu \mathrm{mol} / \mathrm{L}$ solution at room temperature for 10 minutes, followed by washing. Intracellular calcium was measured by exciting cardiomyocytes alternately at 340 and $380 \mathrm{~nm}$ and reading emission at $505 \mathrm{~nm}$. Function was measured by using video-based detection and calculation of sarcomere length (IonOptix, Milton, Mass). Unloaded cells were paced (MyoPacer, IonOptix) to contract at a frequency of $1 \mathrm{~Hz}$ while sarcomere length and Fura-2 transients were recorded. A minimum of 10 transients were averaged from each cell and treatment. Measures of sarcomere length and intracellular calcium analyzed included resting (diastolic) and peak (systolic) values, maximum departure and return velocities, the time of transient onset, peak time, and the constant tau describing calcium removal. Cells were excluded from analysis if they displayed membrane blebs, resting sarcomere lengths of less than $1.6 \mu \mathrm{m}$, frequent spontaneous contractions without stimulation, or less than $5 \%$ shortening.

\section{Isolated Cardiomyocyte Experimental Protocols}

Cells were separated into 3 treatment groups. The numbers of cardiomyocytes per group ranged from 9 to 21 and were derived from 4 to 6 individual rats. Each treatment group consisted of a baseline, test, and recovery phase, with functional parameters measured at the end of each phase. For swelling studies, cells were first perfused with LowNa Isotonic buffer, followed by 8 minutes in $0.75 \mathrm{~T}$ buffer and recovery in LowNa Isotonic buffer for 8 minutes. Control cells were perfused continuously with LowNa Isotonic buffer, and recordings were made at times equivalent with the test group. In ischemic experiments cells were perfused with Isotonic buffer followed by 10 minutes in ischemic buffer. Recovery was measured at 3 and 10 minutes of reperfusion in either LowNa Isotonic buffer or $0.75 \mathrm{~T}$ buffer.

Cell area was calculated for all cells by using images captured at the end of each treatment period (baseline, test, and recovery) of cardiomyocytes in diastole. Cell borders were manually traced, and area in square micrometers was calculated with ImageJ software (National Institutes of Health, Bethesda, Md).

\section{Whole-Heart Functional Analysis}

Hearts were removed following the same procedure as for preparation of isolated cardiomyocytes, cannulated, and perfused through a Langendorff apparatus (Radnoti Glass Technology, Inc, Monrovia, Calif) at 80 to 90 $\mathrm{mm} \mathrm{Hg}$ of pressure with carbogen $\left(95 \% \mathrm{O}_{2}\right.$ and $\left.5 \% \mathrm{CO}_{2}\right)$ gassed buffers warmed to $37^{\circ} \mathrm{C}$, as previously described. ${ }^{15}$ The standard Krebs buffer consisted of $118 \mathrm{mmol} / \mathrm{L} \mathrm{NaCl}, 25 \mathrm{mmol} / \mathrm{L} \mathrm{NaHCO}_{3}, 4.8 \mathrm{mmol} / \mathrm{L} \mathrm{KCl}, 1.2$ $\mathrm{mmol} / \mathrm{L} \mathrm{KH}_{2} \mathrm{PO}_{4}, 1.2 \mathrm{mmol} / \mathrm{L} \mathrm{MgSO}_{4}, 1.2 \mathrm{mmol} / \mathrm{L} \mathrm{CaCl}_{2}$, and glucose. ${ }^{11}$ The left atrial appendage was removed, and a drain was inserted to prevent fluid build-up in the left ventricle. A latex balloon was then placed in the left ventricle, and diastolic pressure was set to $5 \mathrm{~mm} \mathrm{Hg}$. Measurements of left ventricular pressure (systolic and diastolic) and heart rate were collected online in real time (PowerLab; ADInstruments, Inc, Colorado Springs, Colo). Individual hearts were only included if the intrinsic heart rate was greater than 200 beats/min and achieved greater than $100 \mathrm{~mm} \mathrm{Hg}$ systolic pressure within 20 minutes of perfusion.

\section{Whole-Heart Experimental Protocols}

Control nonperfused hearts were removed as for cannulated hearts but immediately processed without perfusion. For all other treatment groups, hearts were first stabilized for 20 minutes. Two main experimental designs were used to investigate the effect of (1) osmotic or oncotic pressure changes and (2) ischemia. Mild osmotic and oncotic changes were produced according to the methodology of Kellen and Bassingthwaighte. ${ }^{16}$ Hearts were perfused with Krebs buffer plus an additional infusion of $15 \mathrm{mmol} /$ $\mathrm{L} \mathrm{NaCl}, 30 \mathrm{mmol} / \mathrm{L}$ sucrose, or $0.5 \%$ dextran. Severe hypotonicity was achieved by removing $35 \mathrm{mmol} / \mathrm{L} \mathrm{NaCl}$ from the Krebs buffer. This produced a $0.75 \mathrm{~T}$ hypotonic equivalent to match that used in cardiomyocyte experiments. Infusion of $70 \mathrm{mmol} / \mathrm{L}$ sucrose to the $0.75 \mathrm{~T}$ Krebs buffer produced isosmotic Krebs buffer (see Table E1). For ischemia experiments, hearts were perfused with Krebs buffer. Baseline function was measured for 20 minutes, followed by 30 minutes of zero-flow ischemia and 50 minutes of reperfusion. These were matched by a perfusion control group, which was produced by maintaining perfusion for 100 minutes. At the end of perfusion, all hearts were weighed and dried before reweighing to determine the percentage water by using the following formula:

$$
\text { (Weight }[\text { Wet }]-\text { Weight }[\text { Dry }] / \text { Weight }[\text { Wet }]) \times 100 \text {. }
$$

\section{Statistical Analysis}

Results are expressed as means \pm standard error of the mean. All statistical analyses were performed with SPSS version 15.0 (SPSS, Inc, Chicago, Ill). For isolated myocyte and heart water analyses, the Kruskal-Wallis test, followed by the Mann-Whitney $U$ test, was used to compare treatment and control groups. Within-group comparison of baseline, test, and recovery values used the Friedman test, followed by the Wilcoxon signed-rank test. In isolated 
cardiomyocyte analyses, significance was only accepted if both within-group and between-group significance from the isotonic control group were shown to account for variability in data and time-dependent changes in cardiomyocyte function. Sequential time-based measurements of function obtained from Langendorff experiments were analyzed with analysis of variance for repeated measures followed by least significant difference post-hoc testing.

\section{RESULTS}

\section{Effect of Cardiomyocyte Swelling on Function}

Mean cardiomyocyte area after test and recovery perfusion were measured. Cardiomyocyte cell size was not significantly changed during continuous perfusion in isotonic buffer. Hypotonic exposure resulted in swelling to $7.0 \%$ greater than baseline $(P<.001)$. Reperfusion with isotonic buffer resulted in a return of cardiomyocyte size to just below the baseline value $(P<.05)$.

Transient data for all isolated cardiomyocyte treatment groups can be found in Table E2. Calcium transients of cardiomyocytes were altered by swelling. Although the resting level of intracellular calcium was unchanged (Figure 1, A), peak intracellular calcium levels were lower in swollen cardiomyocytes relative to baseline $(P<.001)$ and isotonic control $(P=.001)$ values (Figure $1, B)$. Other aspects of intracellular calcium dynamics were unchanged, including calcium clearance (Figure 1, C).

The only statistically significant effect of swelling on cardiomyocyte shortening was a small decrease in the time to
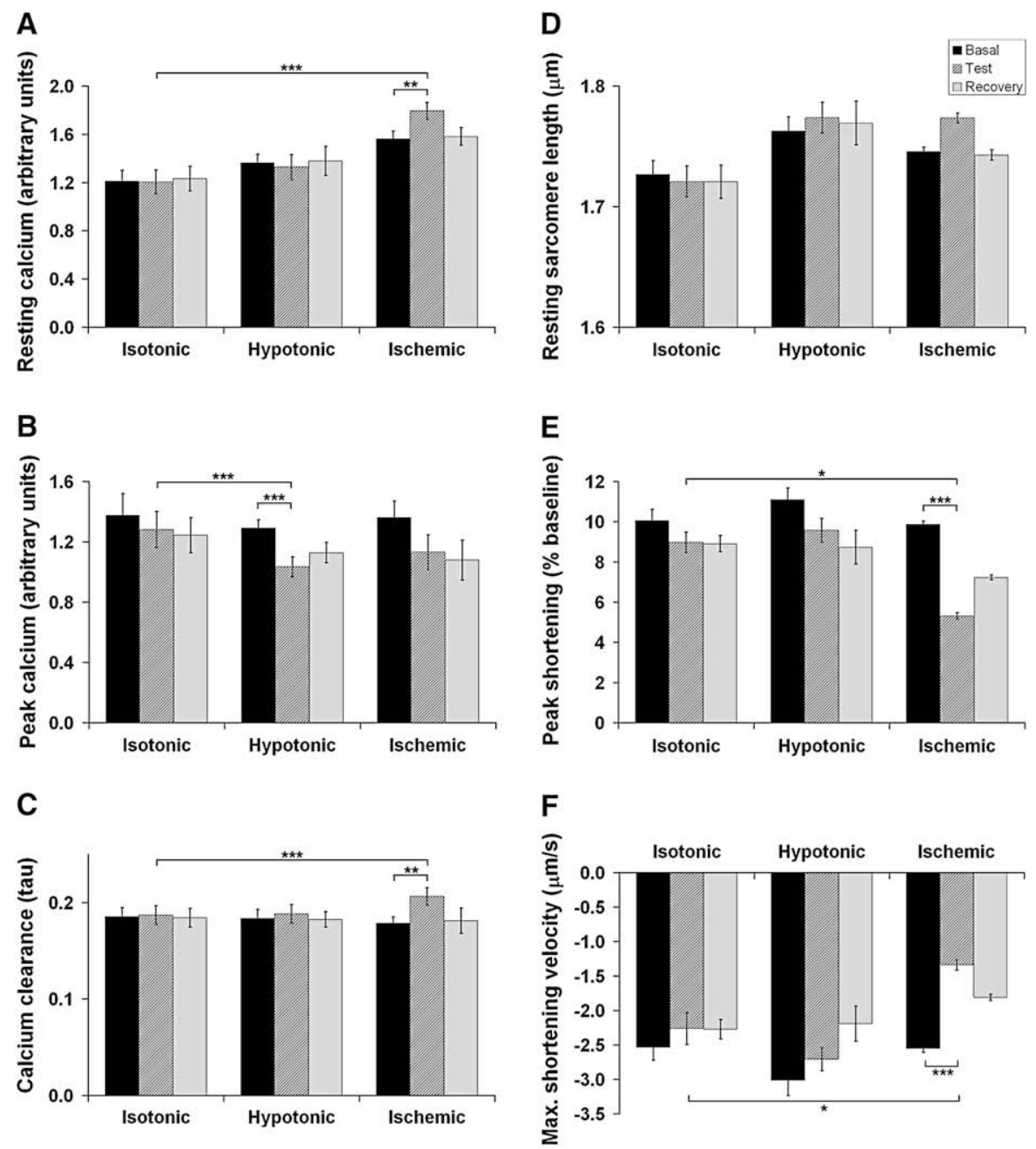

FIGURE 1. Mean transient data are shown for intracellular calcium (left panel) and sarcomere shortening (right panel) of isolated cardiomyocytes. Measurements were made at baseline (black bars), test (hatched bars), and recovery (gray bars). The isotonic control group was produced by continuous perfusion of cells with isotonic buffer and used for statistical comparison with the hypotonic and ischemic test groups. A, Diastolic intracellular calcium values are significantly increased in ischemic cells $(* * * P<.001$ isotonic vs ischemic; $* * P<.01$ basal vs ischemic) and returned to the baseline level with isotonic reperfusion. B, Peak intracellular calcium values are reduced in swollen cardiomyocytes of the hypotonic group (***P<.001 isotonic vs hypotonic; $* * * P<.001$ basal vs hypotonic). C, The calcium clearance constant tau is significantly increased during ischemic exposure (*** $P<.001$ isotonic vs ischemic; $* * P<.01$ basal vs ischemic). D, Diastolic sarcomere length is not significantly altered by hypotonic or ischemic treatment. E, Peak shortening is significantly reduced by ischemia $(* P<.05$ isotonic vs ischemic; $* * * P<.001$ basal vs ischemic). $\mathrm{F}$, Maximum velocity of shortening is significantly reduced by ischemia $(* P<.05$ isotonic vs ischemic; $* * * P<.001$ basal vs ischemic). Data are presented as means \pm standard error of the mean. 
peak shortening $(P<.01$, test vs baseline; $P<.05$, hypotonic vs isotonic control). Resting sarcomere length (Figure $1, D$ ) was unchanged by hypotonic challenge. Despite the reduced peak calcium value seen in swollen cardiomyocytes, peak shortening was not significantly different from that seen in isotonic control specimens (Figure 1,E).

\section{Effect of Cardiomyocyte Ischemia on Function}

Ischemia resulted in an average $2.3 \%$ increase in cardiomyocyte volume relative to baseline values $(P<.01)$, with return to normal size after isotonic recovery. When ischemic cells were recovered in $0.75 \mathrm{~T}$ buffer, area increased further to $5.8 \%$ greater than the baseline value. This was statistically significant when compared with both baseline $(P<$ $.01)$ and ischemic $(P<.01)$ cardiomyocyte size.

Cardiomyocytes subjected to ischemia displayed increased intracellular calcium in diastole compared with within-group baseline values $(P<.01)$ and the isotonic control group $(P=.001$; Figure $1, A)$, which resolved with isotonic recovery. Peak calcium values were unchanged (Figure 1, B). Slower calcium clearance was seen in ischemic cells as an increase in tau compared with the baseline value $(P<.01)$ and as a relative change compared with that seen in the isotonic control group $(P<.001$; Figure 1, C).

Ischemic insult profoundly affected cardiomyocyte shortening. Ischemic cells displayed a small increase in diastolic sarcomere length $(P<.01$; Figure $1, D)$ and reduced velocity of relengthening $(P<.01)$ compared with their baseline value that was not significantly different from that of the isotonic control specimens. Peak shortening was greatly reduced in ischemic cells $(P=.001$, test vs baseline; $P<$ .05 , ischemic vs isotonic control; Figure $1, E)$. Maximum shortening velocity was also impaired $(P=.001$, test vs baseline; $P<.05$, ischemic vs isotonic control; Figure 1, $F)$. A small reduction in the time to peak shortening of ischemic cardiomyocytes $(P<.01$, test vs baseline; $P<.01$, ischemic vs isotonic control) returned to baseline with isotonic recovery.

\section{Langendorff Perfusion and Heart Water Content}

Langendorff-perfused hearts became swollen in comparison with nonperfused hearts, taking on $6.2 \%$ more water. For the group sizes used, water content was not measurably affected by small increases in osmotic or oncotic balance induced by infusion of $15 \mathrm{mmol} / \mathrm{L} \mathrm{NaCl}, 30 \mathrm{mmol} / \mathrm{L}$ sucrose, or $0.5 \%$ dextran when compared with control Krebsperfused hearts (data not shown). Water loading was also unchanged at the end of ischemia and reperfusion compared with that seen in control-perfused hearts (data not shown).

Hyposmotic perfusion with $0.75 \mathrm{~T}$ Krebs buffer induced swelling of Langendorff-perfused hearts. For comparison with later functional data, water content of a subset of hearts $(n=4$ per group) was compared at the end of each perfusion phase: after 20 minutes of isosmotic Krebs perfusion, after hypotonic Krebs perfusion, and after reperfusion in isosmotic Krebs buffer. Hearts perfused with $0.75 \mathrm{~T}$ hyposmotic Krebs buffer took on 3.3\% more water than hearts perfused with isosmotic Krebs buffer $(P<.05)$. This swelling resolved to baseline values during isosmotic recovery.

\section{Langendorff Perfusion and Functional Analysis}

Systolic and diastolic pressures of the main experimental groups are shown in Figure 2. Diastolic pressure was unaffected by continuous perfusion in isosmotic Krebs buffer, whereas a nonsignificant trend toward decreasing systolic pressure was seen over time (data not shown). The functional effect of small osmotic or oncotic increases was measured with infusion of $15 \mathrm{mmol} / \mathrm{L} \mathrm{NaCl}, 30 \mathrm{mmol} / \mathrm{L}$ sucrose, or $0.5 \%$ dextran. Systolic and diastolic pressures were not statistically altered by infusion with $\mathrm{NaCl}$ (Figure 2, A) or sucrose. Diastolic pressure in the presence of $0.5 \%$ dextran infusion was significantly higher than without this oncotic agent $(P<.01$; Figure $2, B)$. Systolic pressure was unaffected (Figure 2, $B$ ).

Diastolic pressure was mildly but significantly increased when the perfusate was changed from isosmotic to $0.75 \mathrm{~T}$ hyposmotic $(P<.001)$ and returned to baseline during subsequent perfusion in isosmotic Krebs buffer (Figure 2, $C$ ). Systolic pressure was stably reduced during $0.75 \mathrm{~T}$ hyposmotic perfusion $(P<.01)$ and also recovered to control values with return to isosmotic perfusion (Figure 2, $C$ ).

Injury induced by 30 minutes of room-temperature global ischemia produced diastolic dysfunction, which was seen as an increase in diastolic pressure $(P<.05$, reperfusion vs baseline; Figure 2, $D$ ). Systolic pressure was decreased in the reperfusion phase compared with the baseline value $(P<.05$, reperfusion vs baseline; Figure $2, D)$ but was not statistically significant from that seen in control-perfused hearts (data not shown).

\section{DISCUSSION}

The primary aim of this study was to investigate the effect of edema on cardiomyocyte and whole-heart function. We used models to analyze the effect of edema without other insults that confound previous studies in this area. Separately, we subjected isolated cardiomyocytes and whole hearts to ischemic injury. Ischemia is a necessary aspect of cardiac surgery involving cardiopulmonary bypass, and our investigations are performed with a long-term view toward improving clinical treatment in this area.

\section{Edema and Cardiac Function}

Our data show a temporal correlation that supports a modest but causal link between edema and dysfunction. Although we provide support for this relationship, it is not as striking as has been suggested by others. Work by Mehlhorn and colleagues ${ }^{3}$ using surgical models has shown a $3 \%$ 

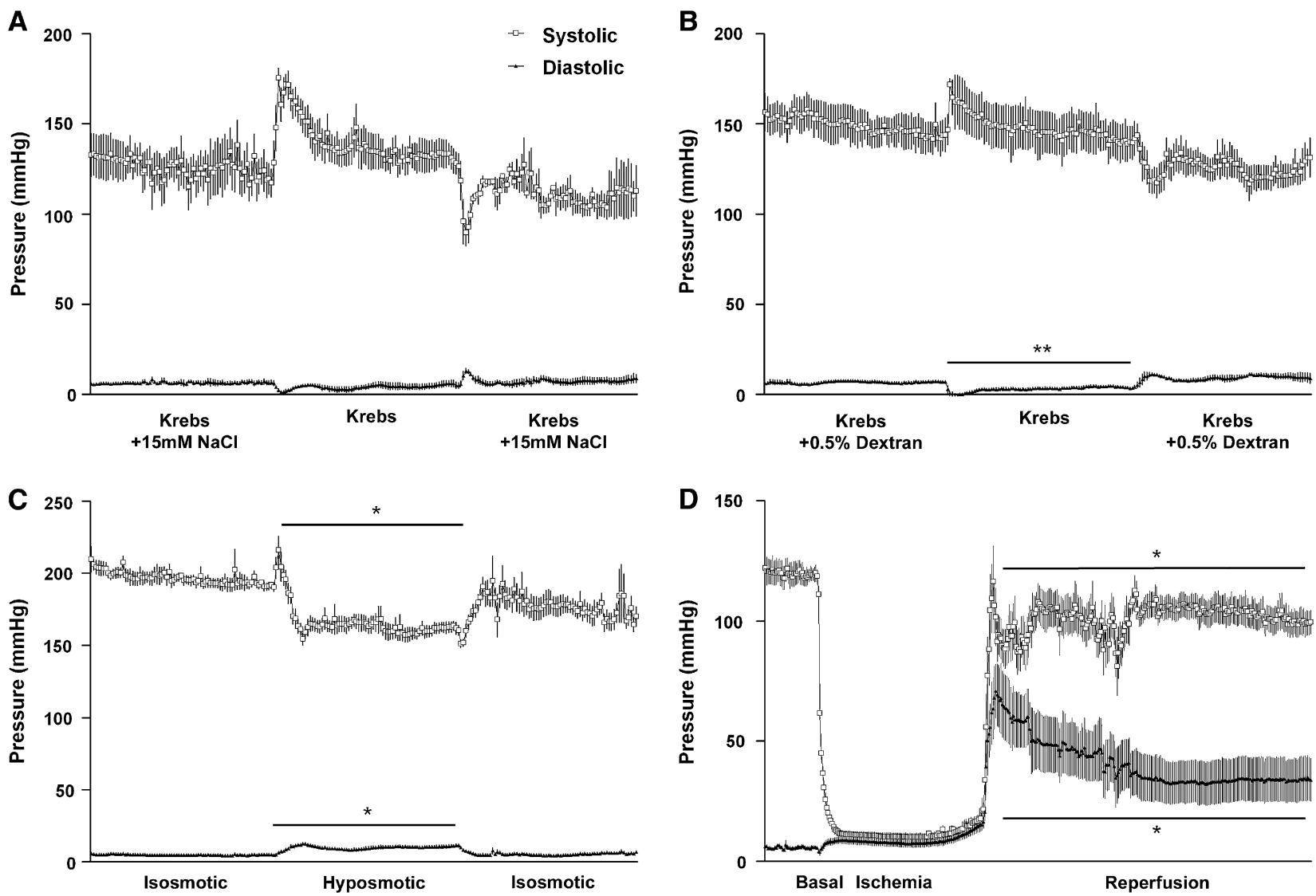

FIGURE 2. Mean systolic and diastolic pressures throughout perfusion. For experiments using osmotic/oncotic change by infusion (A-C) the experiment was 60 minutes total, made up of 20 minutes with infusion, 20 minutes without infusion, and 20 minutes with infusion. A, $\pm 15 \mathrm{mmol} / \mathrm{L} \mathrm{NaCl}$. No significant differences were seen. $\mathrm{B}, \pm 0.5 \%$ dextran. Diastolic pressure decreased when dextran infusion was stopped $(* * P<.01)$. C, $0.75 \mathrm{~T}$ hyposmotic Krebs buffer made isosmotic with $70 \mathrm{mmol} / \mathrm{L}$ sucrose infusion. Systolic $(* P<.05)$ and diastolic $(* P<.05)$ pressures of swollen hearts in the $0.75 \mathrm{~T}$ hyposmotic phase were significantly different from both baseline and recovery isosmotic values. D, For ischemic experiments, 10 minutes of baseline measurement is followed by 30 minutes of no-flow ischemia in which systolic pressure decreased to near zero and 50 minutes of reperfusion. Systolic $(* P<.05)$ and diastolic $(P<.05)$ pressures in the reperfusion phase are significantly different from baseline. Note that the time course and scale in panels $\mathrm{C}$ and $\mathrm{D}$ are different and should not be directly compared. Shown are means \pm standard error of the mean.

increase in heart water content to be associated with as much as a $50 \%$ decrease in cardiac function. Langendorff-based investigation has suggested a $5 \%$ increase in cardiac water to be sufficient to inhibit developed pressure by $50 \% .^{17}$ Our data show that small differences in osmotic or oncotic pressure, which are known to alter water content, ${ }^{16}$ were tolerated by Langendorff-perfused hearts, with no deleterious effect on function. Swelling of $3.3 \%$ greater than that seen with control-perfused hearts was associated with a reduction in developed pressure of $9 \%$ that resolved completely on return to baseline water content. Other investigators have suggested that small increases in water content might not affect cardiac function, ${ }^{18}$ and we conclude that even moderate increases are well tolerated.

In the clinical setting cardiac edema after cardiopulmonary bypass is largely thought to be caused by interstitial water accumulation. The effect of interstitial edema on cardiac performance was not examined because of the technical con- straints of our ex vivo models. Tissue water increases during early equilibration of Langendorff perfusion, ${ }^{19}$ which is likely to be interstitial water gain. Reliable functional measurements do not begin until after equilibration, and as such, both the control and treatment groups are similarly swollen initially. Hyposmotic perfusion after equilibration results in increased intracellular water. ${ }^{20}$ As such, our Langendorff model tests the functional effect of intracellular swelling.

Intracellular edema induced in whole hearts resulted in reduced developed pressure, as has been seen by others, ${ }^{21}$ although isolated cardiomyocyte shortening was unaffected by swelling. The functional deficit seen in swollen Langendorff-perfused hearts might be explained by impaired force development; swelling of isolated cardiomyocytes was associated with reduced peak intracellular calcium values, and this is also likely to occur in whole hearts. Regardless of the cause of the dysfunction, our data suggest that 
intracellular water must be investigated in a whole-organ setting to provide clinically useful information.

\section{Ischemia and Cardiac Function}

Modeling ischemia in vitro is necessarily different from in vivo cardiac ischemia induced in the context of cardiopulmonary bypass. In particular, stopping the heart with cardioplegia, the addition of various substrates, and cooling during surgical intervention aims to minimize ischemic injury. We modeled ischemia in isolated cardiomyocytes with a buffer that mimics the ischemic milieu, including the presence of lactate, but maintained continuous cardiomyocyte contraction. The whole-heart Langendorff model produced global ischemia also with continuous contraction. We recognize that these models are fundamentally different from the surgical situation. However, they allow us to clarify the specific effect of ischemia without confounders, such as hypothermia or cardioplegia. Furthermore, comparison can be made between our results and those of other researchers, ${ }^{14,22,23}$ validating the model and thus comparison of edematous and ischemic effects.

Cardiac edema and dysfunction have been linked in the context of cardioplegia studies. In some instances dysfunction has been attributed to edema without controlling for the effects of hypothermia and hyperkalemia. ${ }^{6}$ Mild hypothermia in anesthetized dogs induces diastolic dysfunction. ${ }^{24}$ Support for hyperkalemia as a primary effector is provided by demonstration of improved systolic function of isolated hearts after ischemia with reduced-potassium cardioplegia ${ }^{10}$ and normokalemic cardioplegia ${ }^{25}$ in comparison with a standard hyperkalemic cardioplegia. The potential additive effect of multiple injuries in these models is highlighted, including the finding that cardioplegia itself induces edema. We have examined these issues in a separate model of infant cardiac surgery. ${ }^{7}$

Global ischemic injury in Langendorff experiments induced ongoing functional impairment in the recovery phase. Ischemic injury in isolated cardiomyocytes aimed to replicate the injury of the whole-heart model as closely as possible. The lactate concentration in particular was chosen to induce functional impairment but not cell death and is within the physiologic range reported during ischemia in vivo. ${ }^{4,26}$ The severity of injury induced was broadly similar in the 2 models. In the recovery phase after ischemia, peak shortening of isolated cardiomyocytes recovered to $84 \%$ of control values, and Langendorff-perfused hearts generated developed pressures that were $75 \%$ of control values.

Tissue osmolarity is expected to increase during cardiac ischemia, resulting in an osmotic imbalance with reperfusion that induces swelling. Although we saw no difference between ischemic and control hearts in water content, others have shown edema in the reperfusion phase after global ischemia. ${ }^{23}$ Osmotic fragility resulting from ischemic injury might contribute to postischemic swelling and functional decline. How- ever, our comparison of ischemic cardiomyocyte function and calcium dynamics when recovered in either isotonic or $0.75 \mathrm{~T}$ hypotonic buffer revealed no differences (data not shown). Using the Langendorff model, others have demonstrated ischemia-induced membrane permeability that protects cardiomyocytes from swelling in the reperfusion phase. ${ }^{20}$ Further work is needed to determine whether edema might act as a significant cocontributor to ischemia-reperfusion injury.

\section{CONCLUSIONS}

Ischemia, as expected, directly affects the cardiomyocyte. Sustained functional deficits are seen in both isolated cells and whole hearts injured by ischemia. Reduced systolic pressures in whole edematous hearts might be related to the reduced peak calcium values seen in swollen cardiomyocytes. The functional inhibition is transient and does not extend beyond the phase of swelling.

Reducing the morbidity that can result from pediatric cardiac surgery depends on better myocardial management. A clear understanding of the causes of myocardial dysfunction is fundamental to this aim. When studied in isolation, our data argue against edema as a major contributor to reduced cardiac performance. Edema might exacerbate dysfunction in the context of insults such as ischemic damage but further modeling is necessary to determine the extent to which this contributes.

We thank Prof John Headrick, Dr Melissa Reichelt, and Prof David Allen for advice and assistance in establishing the models used in this manuscript. We thank Joanne Hawkes for excellent technical assistance.

\section{References}

1. Butler J, Rocker GM, Westaby S. Inflammatory response to cardiopulmonary bypass. Ann Thorac Surg. 1993;55:552-9.

2. Anselmi A, Abbate A, Girola F, Nasso G, Biondi-Zoccai GG, Possati G, et al. Myocardial ischemia, stunning, inflammation, and apoptosis during cardiac surgery: a review of evidence. Eur J Cardiothorac Surg. 2004;25:304-11.

3. Mehlhorn U, Geissler HJ, Laine GA, Allen SJ. Myocardial fluid balance. Eur J Cardiothorac Surg. 2001;20:1220-30.

4. Tranum-Jensen J, Janse MJ, Fiolet WT, Krieger WJ, D'Alnoncourt CN, Durrer D. Tissue osmolality, cell swelling, and reperfusion in acute regional myocardial ischemia in the isolated porcine heart. Circ Res. 1981;49:364-81.

5. Seghaye MC. The clinical implications of the systemic inflammatory reaction related to cardiac operations in children. Cardiol Young. 2003;13:228-39.

6. Mizutani S, Al-Dadah AS, Bloch JB, Prasad SM, Diodato MD, Schuessler RB, et al. Hyperkalemic cardioplegia-induced myocyte swelling and contractile dysfunction: prevention by diazoxide. Ann Thorac Surg. 2006;81:154-9.

7. Egan JR, Butler TL, Cole AD, Aharonyan A, Baines D, Street N, et al. Myocardial ischemia is more important than the effects of cardiopulmonary bypass on myocardial water handling and postoperative dysfunction: a pediatric animal model. $J$ Thorac Cardiovasc Surg. 2008;136:1265-73.

8. Li GR, Zhang M, Satin LS, Baumgarten CM. Biphasic effects of cell volume on excitation-contraction coupling in rabbit ventricular myocytes. Am J Physiol Heart Circ Physiol. 2002;282:H1270-7.

9. Al-Dadah AS, Voeller RK, Schuessler RB, Damiano RJ Jr, Lawton JS. Maintenance of myocyte volume homeostasis during stress by diazoxide is cardioprotective. Ann Thorac Surg. 2007;84:857-62.

10. Jayawant AM, Stephenson ER Jr, Baumgarten CM, Damiano RJ Jr. Prevention of cell swelling with low chloride St. Thomas' Hospital solution improves postischemic myocardial recovery. J Thorac Cardiovasc Surg. 1998;115:1196-202. 
11. Ogura T, Matsuda H, Imanishi S, Shibamoto T. Sarcolemmal hydraulic conductivity of guinea-pig and rat ventricular myocytes. Cardiovasc Res. 2002;54: 590-600.

12. Maehara T, Novak I, Wyse RK, Elliot MJ. Perioperative monitoring of total body water by bio-electrical impedance in children undergoing open heart surgery. Eur J Cardiothorac Surg. 1991;5:258-65.

13. Wernovsky G, Wypij D, Jonas RA, Mayer JE Jr, Hanley FL, Hickey PR, et al. Postoperative course and hemodynamic profile after the arterial switch operation in neonates and infants. A comparison of low-flow cardiopulmonary bypass and circulatory arrest. Circulation. 1995;92:2226-35.

14. Lu J, Zang WJ, Yu XJ, Chen LN, Zhang CH, Jia B. Effects of ischaemia-mimetic factors on isolated rat ventricular myocytes. Exp Physiol. 2005;90:497-505.

15. Butler TL, Au CG, Yang B, Egan JR, Tan YM, Hardeman EC, et al. Cardiac aquaporin expression in humans, rats, and mice. Am J Physiol Heart Circ Physiol. 2006;291:H705-13.

16. Kellen MR, Bassingthwaighte JB. Transient transcapillary exchange of water driven by osmotic forces in the heart. Am J Physiol Heart Circ Physiol. 2003; 285:H1317-31.

17. Rubboli A, Sobotka PA, Euler DE. Effect of acute edema on left ventricular function and coronary vascular resistance in the isolated rat heart. Am J Physiol Heart Circ Physiol. 1994;267:H1054-61.

18. Fischer UM, Cox CS Jr, Stewart RH, Laine GA, Allen SJ. Impact of acute myocardial edema on left ventricular function. J Invest Surg. 2006;19:31-8.
19. Aliev MK, Dos Santos P, Hoerter JA, Soboll S, Tikhonov AN, Saks VA. Water content and its intracellular distribution in intact and saline perfused rat hearts revisited. Cardiovasc Res. 2002;53:48-58.

20. Askenasy N, Vivi A, Tassini M, Navon G, Farkas DL. NMR spectroscopic characterization of sarcolemmal permeability during myocardial ischemia and reperfusion. J Mol Cell Cardiol. 2001;33:1421-33.

21. Geissler HJ, Allen SJ. Myocardial fluid balance: pathophysiology and clinical implications. J Thorac Cardiovasc Surg. 1998;46:242-7.

22. Maddaford TG, Hurtado C, Sobrattee S, Czubryt MP, Pierce GN. A model of lowflow ischemia and reperfusion in single, beating adult cardiomyocytes. Am J Physiol Heart Circ Physiol. 1999;277:H788-98.

23. Palmer BS, Hadziahmetovic M, Veci T, Angelos MG. Global ischemic duration and reperfusion function in the isolated perfused rat heart. Resuscitation. 2004;62: 97-106.

24. Fischer UM, Cox CS Jr, Laine GA, Mehlhorn U, Allen SJ. Mild hypothermia impairs left ventricular diastolic but not systolic function. J Invest Surg. 2005;18 291-6.

25. Dobson GP, Jones MW. Adenosine and lidocaine: a new concept in nondepolarizing surgical myocardial arrest, protection, and preservation. J Thorac Cardiovasc Surg. 2004;127:794-805.

26. Kennergren C, Mantovani V, Strindberg L, Berglin E, Hamberger A, Lonnroth P. Myocardial interstitial glucose and lactate before, during, and after cardioplegic heart arrest. Am J Physiol Endocrinol Metab. 2003;284:E788-94. 
TABLE E1. Primary buffer components for isolated cardiomyocytes and Langendorff-perfused hearts

\begin{tabular}{llll} 
Osmolarity (mOsm approximation) & $\mathrm{NaCl}(\mathrm{mmol} / \mathrm{L})$ & Sucrose $(\mathrm{mmol} / \mathrm{L})$ & Lactate $(\mathrm{mmol} / \mathrm{L})$ \\
\hline
\end{tabular}

Ogura Tyrode's buffer: isolated cardiomyocytes

Isotonic $(\mathrm{pH} 7.4)$

$0.75 \mathrm{~T}$ Hypotonic $(\mathrm{pH} 7.4)$

LowNa Isotonic ( $\mathrm{pH} 7.4)$

Ischemic (pH 6.8)

Krebs buffer: Langendorff-perfused hearts

Isosmotic Krebs buffer

0.75T Hyposmotic Krebs buffer

LowNa Isosmotic Krebs buffer

$\begin{array}{lr}320 & 14 \\ 240 & 10 \\ 320 & 10 \\ 320 & 140 \\ & \\ 330 & 11 \\ 260 & 83 \\ 330 & 83\end{array}$

$\begin{array}{rr}140 & - \\ 105 & - \\ 105 & 70 \\ 140 & - \\ 118 & - \\ 83 & - \\ 83 & 70\end{array}$

\begin{tabular}{cc}
- & - \\
- & - \\
- & - \\
70 & - \\
- & 2.5 \\
& \\
- & - \\
- & - \\
70 & - \\
\hline
\end{tabular}

TABLE E2. Shortening and Fura-2 transients for isolated cardiomyocytes

\begin{tabular}{|c|c|c|c|c|c|c|c|c|c|}
\hline & & \multicolumn{4}{|c|}{ Shortening transient } & \multicolumn{4}{|c|}{ Calcium transient } \\
\hline & & Group A & Group B & Group C & Group D & Group A & Group B & Group C & Group D \\
\hline \multirow[t]{3}{*}{ Resting } & Baseline & $1.727 \pm 0.015$ & $1.763 \pm 0.012$ & $1.742 \pm 0.017$ & $1.749 \pm 0.017$ & $1.213 \pm 0.064$ & $1.364 \pm 0.088$ & $1.577 \pm 0.100$ & $1.543 \pm 0.102$ \\
\hline & Test & $1.721 \pm 0.016$ & $1.774 \pm 0.013$ & $1.776 \pm 0.019$ & $1.771 \pm 0.018$ & $1.206 \pm 0.070$ & $1.328 \pm 0.099$ & $1.798 \pm 0.148 \dagger$ & $1.791 \pm 0.147$ \\
\hline & Recovery & $1.721 \pm 0.015$ & $1.769 \pm 0.014$ & $1.743 \pm 0.018$ & $1.738 \pm 0.020$ & $1.233 \pm 0.073$ & $1.380 \pm 0.103$ & $1.581 \pm 0.121$ & $1.714 \pm 0.158$ \\
\hline \multirow{3}{*}{$\begin{array}{l}\text { Dep v } \\
\text { (U/s) }\end{array}$} & Baseline & $-2.532 \pm 0.350$ & $-3.012 \pm 0.193$ & $-2.617 \pm 0.292$ & $-2.470 \pm 0.353$ & $30.723 \pm 2.546$ & $28.954 \pm 3.301$ & $28.282 \pm 1.438$ & $31.498 \pm 2.113$ \\
\hline & Test & $-2.261 \pm 0.314$ & $-2.707 \pm 0.234$ & $-1.302 \pm 0.250 *$ & $-1.385 \pm 0.229$ & $28.788 \pm 3.122$ & $23.743 \pm 2.761$ & $23.156 \pm 1.340$ & $23.990 \pm 3.042$ \\
\hline & Recovery & $-2.272 \pm 0.329$ & $-2.190 \pm 0.141$ & $-1.808 \pm 0.256$ & $-2.234 \pm 0.274$ & $26.833 \pm 3.366$ & $24.914 \pm 2.823$ & $23.140 \pm 2.118$ & $21.522 \pm 2.171$ \\
\hline \multirow{3}{*}{$\begin{array}{c}\% \text { Peak h/ } \\
\text { peak h }\end{array}$} & Baseline & $10.071 \pm 1.130$ & $11.107 \pm 0.550$ & $9.571 \pm 0.765$ & $10.230 \pm 0.927$ & $1.376 \pm 0.110$ & $1.291 \pm 0.145$ & $1.289 \pm 0.071$ & $1.461 \pm 0.079$ \\
\hline & Test & $8.983 \pm 0.960$ & $9.572 \pm 0.500$ & $4.852 \pm 0.848^{*}$ & $5.898 \pm 0.831$ & $1.283 \pm 0.116$ & $1.035 \pm 0.119 \ddagger$ & $1.076 \pm 0.073$ & $1.210 \pm 0.120$ \\
\hline & Recovery & $8.915 \pm 0.981$ & $8.738 \pm 0.408$ & $7.239 \pm 0.834$ & $9.259 \pm 0.943$ & $1.245 \pm 0.133$ & $1.129 \pm 0.117$ & $1.080 \pm 0.069$ & $1.031 \pm 0.063$ \\
\hline \multirow[t]{3}{*}{ Peak t (s) } & Baseline & $0.173 \pm 0.006$ & $0.161 \pm 0.007$ & $0.151 \pm 0.009$ & $0.169 \pm 0.009$ & $0.085 \pm 0.004$ & $0.091 \pm 0.006$ & $0.085 \pm 0.007$ & $0.090 \pm 0.003$ \\
\hline & Test & $0.164 \pm 0.006$ & $0.145 \pm 0.009^{*}$ & $0.125 \pm 0.008$ & $0.146 \pm 0.009$ & $0.085 \pm 0.004$ & $0.080 \pm 0.003$ & $0.083 \pm 0.007$ & $0.108 \pm 0.012$ \\
\hline & Recovery & $0.164 \pm 0.007$ & $0.150 \pm 0.008$ & $0.150 \pm 0.007$ & $0.162 \pm 0.010$ & $0.090 \pm 0.004$ & $0.096 \pm 0.008$ & $0.092 \pm 0.006$ & $0.095 \pm 0.012$ \\
\hline \multirow{3}{*}{$\begin{array}{l}\text { Ret } \mathrm{v} \\
\qquad(\mathrm{U} / \mathrm{s})\end{array}$} & Baseline & $1.673 \pm 0.223$ & $2.472 \pm 0.224$ & $2.120 \pm 0.242$ & $2.217 \pm 0.398$ & $-6.494 \pm 0.765$ & $-6.228 \pm 0.904$ & $-6.612 \pm 0.604$ & $-6.682 \pm 0.269$ \\
\hline & Test & $1.595 \pm 0.222$ & $2.201 \pm 0.213$ & $1.190 \pm 0.257$ & $1.161 \pm 0.179$ & $-5.555 \pm 0.638$ & $-5.287 \pm 0.823$ & $-5.129 \pm 0.254$ & $-4.995 \pm 0.525$ \\
\hline & Recovery & $1.785 \pm 0.328$ & $1.886 \pm 0.144$ & $1.527 \pm 0.249$ & $2.080 \pm 0.324$ & $-5.845 \pm 0.968$ & $-5.788 \pm 0.741$ & $-5.625 \pm 0.475$ & $-5.599 \pm 0.657$ \\
\hline \multirow[t]{3}{*}{ Tau } & Baseline & & & & & $0.186 \pm 0.009$ & $0.184 \pm 0.009$ & $0.175 \pm 0.011$ & $0.184 \pm 0.007$ \\
\hline & Test & & & & & $0.187 \pm 0.010$ & $0.188 \pm 0.010$ & $0.200 \pm 0.013 \dagger$ & $0.215 \pm 0.012$ \\
\hline & Recovery & & & & & $0.184 \pm 0.010$ & $0.183 \pm 0.008$ & $0.181 \pm 0.013$ & $0.182 \pm 0.008$ \\
\hline
\end{tabular}

Shortening transients measure sarcomere length in micrometers. Calcium transients are measured as the 340/380 nm ratio of Fura-2. Resting, Diastolic value; Dep v, departure velocity; \% Peak $h$ (shown for shortening transients), systolic peak value as a percentage of diastolic value; peak $h$ (shown for calcium transients), systolic peak value minus diastolic value; Peak $t$, time of peak; Ret v, maximum relaxation velocity; tau, diastolic single exponential constant. Group A, Isotonic control; Group B, 0.75 T hypotonic; Group C, ischemic (isotonic recovery); Group $D$, ischemic (hypotonic recovery). $* P<.05, \dagger P<.01$, and $\ddagger P<.001$. 